\title{
EXTRAÇÃO DE CONTORNO DE TELHADOS DE EDIFICAÇÕES UTILIZANDO DADOS LASER E IMAGENS AÉREAS
}

\section{EXTRACTION OF BUILDINGS' ROOF CONTOURS USING LASER DATA AND AERIAL IMAGES}

Guilherme Augusto Potje ${ }^{1}$; Mario Augusto Pazoti ${ }^{2}$; Francisco Assis da Silva ${ }^{2}$; Danillo Roberto Pereira ${ }^{1}$; Almir Olivette Artero ${ }^{2}$; Marco Antonio Piteri $^{2}$

\footnotetext{
${ }^{1}$ Faculdade de Informática - FIPP, Universidade do Oeste Paulista - UNOESTE e-mail: gui_potje@hotmail.com, \{mario,chico, danilopereira\}@unoeste.br

${ }^{2}$ Faculdade de Ciências e Tecnologia - FCT, Universidade Estadual Paulista - Unesp e-mail: \{almir, piteri\}@fct.unesp.br
}

RESUMO - As tecnologias atuais para obtenção de dados geográficos estão cada vez mais precisas e possibilitam a captura de informações de uma grande área territorial em pouco tempo como, por exemplo, a varredura a laser. Outra tecnologia que já é utilizada há algum tempo é a imagem digital de alta resolução. Nesse contexto, a extração de feições a partir desses dados tem recebido, nos últimos anos, considerável atenção. Com a combinação de tipos diferentes de dados, é possível melhorar os resultados obtidos a partir da extração de objetos em cenas complexas e, por esse motivo, este trabalho desenvolveu uma ferramenta a fim de automatizar a extração do contorno dos telhados.

Palavras-chave: Extração de Feições; Varredura a LASER; Contorno de Telhados.

ABSTRACT - The current technologies for obtaining spatial data are very accurate, and allow the capture information from a wide geographical area in a short time. An example is the laser scanning and another well-known one is the high-resolution aerial photographs. In this context, the extraction from these data has received considerable attention. With the combination of these different types of data, it is possible to improve the results obtained from the extraction of objects in complex scenes and therefore this study aims to develop a tool that automates the extraction of the roofs' contours.

Keywords: Features Extraction; LASER Scanning; Roof's Contours.

Recebido em: 15/05/2014

Revisado em: 20/08/2014

Aprovado em: 05/09/2014 


\section{INTRODUÇÃO}

A extração automática de telhados dentro do contexto de extração de feições urbanas é um assunto que vem ganhando atenção nos últimos anos, fato evidenciado pela recente e ampla literatura produzida sobre o tema e por sua importância tecnológica, haja vista que na maioria das vezes a extração de feições é realizada manualmente, implicando em gastos excessivos e alto custo do processo de captura dos dados. Os métodos de extração de telhados se diferenciam quanto ao tipo de fonte de dados utilizada, podendo ser imagens aéreas e de satélite, dados de varredura a laser, plantas baixas e outras fontes menos conhecidas que, geralmente, são utilizadas em conjunto para se conseguir melhores resultados (GALVANIN, 2007). Existem alguns tipos de reconstrução que são: a reconstrução poliédrica ou tridimensional do edifício, a reconstrução do contorno do telhado ou a simples segmentação para detectar telhados (MACHADO; MITISHITA, 2006).

Sohn e Dowman (2003) ressalta que a extração de contornos de edifícios é um problema difícil no âmbito do reconhecimento de objetos, o que está relacionado com a complexidade e a variabilidade da cena, sendo necessário recorrer a várias representações da mesma área territorial utilizando recursos diferentes.

A classificação de pontos apenas considerando sua altura não é suficiente para identificar prédios, pois existem, num ambiente urbano, outros objetos com altura igual ou superior, como árvores. A principal dificuldade na identificação de construções reside em excluir a vegetação. Usando somente os dados da varredura a laser, esta tarefa é difícil e muitas vezes impossível, sem ajuda de dados auxiliares. A solução mais interessante, e a tendência verificada no uso de varredura a laser em áreas urbanas, é a integração de dados altimétricos com informações espectrais derivadas de fotografias aéreas ou imagens de satélites de alta resolução espacial (CENTENO; MITISHITA, 2007).

Assim, o objetivo deste trabalho é desenvolver uma ferramenta que a partir dos dados de entrada (dados laser associados às imagens aéreas), retorne ao usuário os pontos correspondentes aos contornos dos telhados de construções. O trabalho facilitará a análise e extração de feições dos dados feita pelo profissional da área de cartografia, que realiza esta tarefa manualmente, e auxiliará na redução de esforços e custos para a conclusão da mesma.

$\mathrm{Na}$ seção 2 são descritos 0 funcionamento e as características da varredura a laser. $\mathrm{Na}$ seção 3 são 
apresentados os trabalhos relacionados à extração de feições urbanas e a respectiva metodologia utilizada. $\mathrm{Na}$ seção 4 são mostrados os passos e procedimentos utilizados no desenvolvimento das funções da ferramenta. A seção 5 apresenta os testes realizados e resultados obtidos.

\section{VARREDURA A LASER}

O sistema de varredura laser denominado LIDAR (Light Detection and Ranging) ou LADAR (Laser Detection and Ranging) é uma tecnologia projetada para medição de coordenadas tridimensionais de pontos em uma superfície. Esse sistema baseia-se na emissão de um pulso laser, sendo que seu funcionamento básico consiste na emissão de um pulso laser em direção a uma superfície alvo, captando a reflexão sobre o objeto atingido, conforme ilustrado na Figura 1. Esse processo é classificado em dois princípios: triangulação e time-of-light (CENTENO; MITISHITA, 2007).

Sistemas que se baseiam no princípio de time-of-light calculam a distância do objeto a partir da medição do tempo entre a emissão e o retorno do pulso de laser.

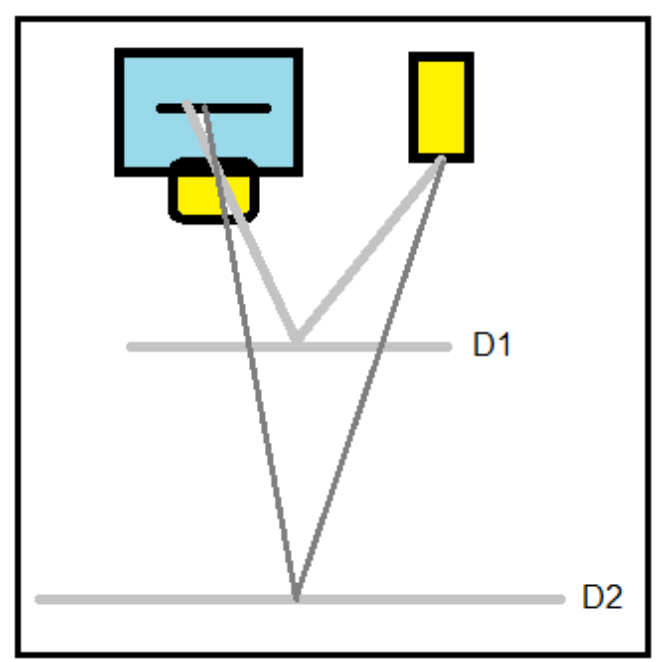

Figura 1. Representação da triangulação traçada a partir do emissor laser, com o ponto da superfície atingida e a câmara.

Primeiramente o pulso de laser é disparado na direção do objeto e uma parte dele é refletida em direção ao sensor. Em seguida, o sensor capta o pulso refletido e realiza medições do tempo decorrido e a intensidade deste pulso. A distância é calculada pela Equação 1, a seguir:

$$
R=c^{*} d t / 2
$$

sendo $R$ a distância entre o sistema de varredura e o objeto, $c$ a velocidade de propagação do raio laser e $d t$ o tempo decorrido entre a emissão e a captação do pulso.

Uma vez coletados os pontos de uma superfície, há a necessidade de representá-lo por meio de uma estrutura de dados, que pode ser classificada em superfície completa e incompleta. Dentre as superfícies incompletas tem-se a Grade Regular, e dentre as completas encontra-se a Rede 
Irregular de Triângulos (TIN), que é uma representação proposta por Peucker, com o intuito de contornar amostragem excessiva ou deficiente proporcionada pelo método da grade regular (BURROUGH, 1989).

A vantagem da Rede Irregular de Triângulos (TIN), é que o espaço de armazenamento necessário é menor, comparado à grade regular, pois o número de pontos mapeados no modelo é pequeno, entretanto, a estrutura de dados para representação de uma rede irregular de triângulos é muito mais complexa que a de uma grade regular, sendo necessária a utilização de algoritmos para a geração e a extração de dados ou feições a partir dos modelos (FAZAN, 2011).

$\mathrm{Na}$ grade regular, o processo para geração consiste em estimar valores de cota de cada ponto da grade a partir de um conjunto de amostras de entrada (FELGUEIRAS, 2001). Para isso, faz-se necessário dividir a nuvem de pontos em setores, na forma de grade, e para cada coordenada dessa grade regular é definida a altura/intensidade com base na ponderação dos pontos vizinhos pertencentes a cada setor. Existem várias técnicas para o cálculo da altura do ponto da malha, sendo o cálculo da mediana entre os pontos dentro do setor uma das mais utilizadas.

Por se assemelhar, em sua forma de representação, aos modelos de dados utilizados no processamento de imagens digitais, as estruturas em grade regular retangular permitem o uso de algoritmos simples para o processamento (FAZAN, 2011), sendo também um dos motivos de se ter adotado essa representação para este trabalho.

A partir dos dados de varredura a laser foram gerados diferentes modelos utilizados neste trabalho. São eles: Modelo Digital de Elevação (MDE), Modelo Digital de Terreno (MDT) e Modelo Digital de Superfície Normalizado (MDSn). Todos esses modelos estão representados em uma grade regular.

O Modelo Digital de Elevação contém informação altimétrica de todos os objetos. Esse modelo é obtido somente com o ajuste da nuvem de pontos em uma grade regular sem nenhum processamento extra. A partir do MDE gera-se o Modelo Digital de Terreno, o qual compreende somente o terreno da região representada, ou seja, desconsideramse os objetos existentes como árvores e construções. A geração desse modelo exige mais processamento, pois além da identificação de pontos que compõem o terreno, são gerados pontos do terreno para as regiões que continham objetos. Por fim, há o Modelo Digital de Superfície Normalizado que contém somente os dados que não estão no nível do terreno. Esse modelo é obtido por meio de uma subtração entre o MDE e o MDT e é o modelo que 
contém os objetos acima do terreno, dentre eles os telhados.

Além da informação altimétrica, outra informação importante contida nos dados laser é a intensidade de retorno. Essa intensidade é diferente para cada tipo de material, o que facilita, em alguns casos, sua identificação. Na Tabela 1, de acordo com Galvanin (2007) tem-se a classificação de alguns materiais de acordo com a intensidade de retorno.

Tabela 1. Percentual de intensidade de reflexão de alguns materiais (GALVANIN, 2007).

\begin{tabular}{|c|c|}
\hline Material & Reflexão(\%) \\
\hline Madeira clara, seca e limpa & 94 \\
\hline Neve & $80-90$ \\
\hline Pedras claras & 85 \\
\hline Calcário, argila & Até 75 \\
\hline Vegetação mista & 60 \\
\hline Coníferas & 30 \\
\hline Asfalto & 17 \\
\hline
\end{tabular}

\section{TRABALHOS RELACIONADOS}

Várias estratégias são abordadas em diferentes trabalhos relacionados à extração de contorno de telhados utilizando dados laser e imagens digitais. Nardinocchi, Forlani e Zingaretti (2003) apresentaram uma estratégia para a reconstrução poliédrica de edifícios isolados utilizando dados de varredura laser. Os autores utilizaram a técnica de crescimento de regiões para obter as bordas da imagem, descreveram as faces planas dos edifícios, e a partir disso uniram as faces para gerar o modelo tridimensional da edificação.

Vosselman e Dijkman (2001) também descreveram uma metodologia para a extração de telhados e geração dos modelos tridimensionais dos edifícios utilizando uma versão tridimensional da transformada de Hough, por meio da detecção das intersecções das faces planas.

Rottensteiner e Briese

desenvolveram um método para a geração automática de modelos tridimensionais de edifícios utilizando um processo de interpolação hierárquica, o qual, a partir de pontos irregularmente distribuídos, classifica os pontos pertencentes ao terreno, edifícios e outras classes de objetos.

Tóvári e Pfeifer (2005) elaboraram uma técnica que combina duas abordagens. A primeira consiste nos critérios geométricos baseados em alturas, inclinações e diferença de curvatura sob a nuvem de pontos, para decidir se o ponto está sobre o terreno ou um objeto. Na segunda abordagem, os dados são segmentados utilizando o algoritmo de crescimento de regiões e, em seguida, é feita uma filtragem tendo como parâmetros critérios de similaridade e distância entre os pontos.

Tarsha-Kurdi et al. (2006) desenvolveram um método automático de segmentação a partir da nuvem de pontos 
(malha irregular de pontos) obtida por varredura a laser usando somente a informação do primeiro pulso laser, com o objetivo de excluir áreas de vegetação.

Destacam-se agora os trabalhos que utilizam a integração duas ou mais fontes de dados para tentar discriminar outros objetos que não façam parte das edificações, com a esperança de se obter melhores resultados.

Park et al. (2006) propuseram um método para extração de edifícios em estruturas complexas de telhados a partir de dados laser e plantas baixas, que consiste na extração de primitivas, e posteriormente na modelagem do edifício, baseada na segmentação de regiões planas do conjunto de pontos fornecidos pela varredura a laser. Esses segmentos planos são usados como primitivas primárias, obtendo assim as primitivas secundárias tais como cumeeiras e fronteiras de beiral para refinar a estrutura dos telhados.

Matikainen, Hyyppä e Hyyppä (2005) utilizaram a integração de dados altimétricos provenientes de varredura a laser com imagens aéreas digitais, auxiliando assim na eliminação de feições irrelevantes, como árvores e sombras no processo de detecção.

Machado e Mitishita (2006) criaram uma metodologia de deteç̧ão automática de contorno de edificações integrando imagens digitais e dados de varredura a laser.
Inicialmente, a imagem é segmentada utilizando o algoritmo de deslocamento pela média, posteriormente, é aplicado o algoritmo de perseguição de contornos para a geração dos contornos das regiões segmentadas. Três filtragens são aplicadas para eliminar regiões não pertencentes a edificações de maneira sucessiva: filtragem para eliminar a vegetação; filtragem para eliminar as regiões baixas; e o filtro DouglasPeucker, que cria feições lineares.

\section{DESENVOLVIMENTO DO PROJETO}

O objetivo deste trabalho é aplicar técnicas de visão computacional para a extração dos contornos dos telhados utilizando como entrada dados laser associadas às imagens aéreas digitais. Uma etapa anterior à extração de contornos de telhados e, que se faz necessária, é a geração de Modelos Digitais de Superfície normalizados (MDSn), resultado proveniente do trabalho de Cardoso, Silva e Pazoti (2014).

Foram realizadas técnicas de filtragem e cálculos sobre esses dados para que forneça como saída os pontos referentes aos contornos dos telhados. A dificuldade em se trabalhar com esses dados se dá tanto pela qualidade de resolução da malha que influencia no resultado final, como na complexidade da cena, neste caso, uma área urbana (com várias edificações de diferentes alturas, muitas áreas arborizadas e, no caso 
específico da imagem digital, várias regiões com sombras).

\subsection{ETAPAS PARA EXTRAÇÃO DOS CONTORNOS DO TELHADO}

O processo de desenvolvimento deste trabalho está representado no fluxograma mostrado na Figura 2. Cada uma das etapas é detalhada nas subseções seguintes.

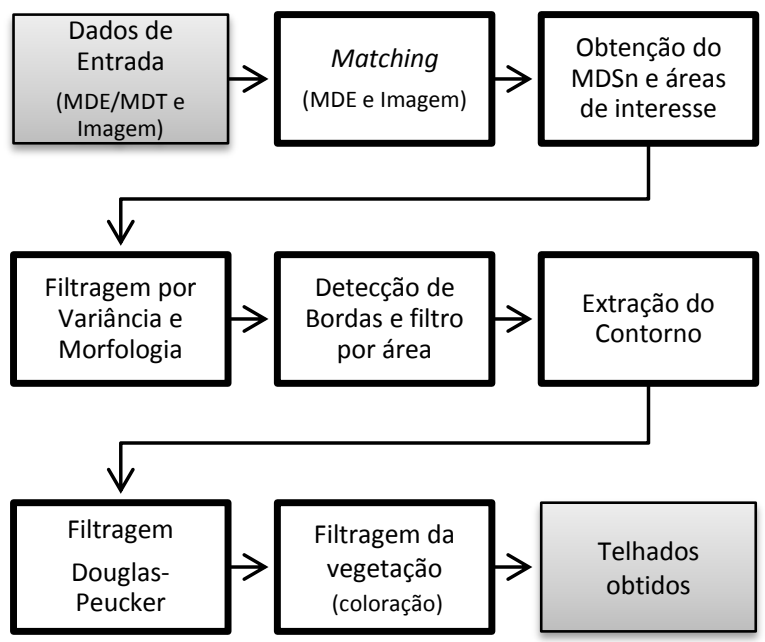

Figura 2. Fluxograma de processos aplicados para realizar a extração do contorno dos telhados.

\subsubsection{MATCHING ENTRE MDE E IMAGEM DIGITAL}

As imagens digitais e os pontos do MDE provenientes da varredura laser não possuem correspondência, pois não houve previamente nenhum tratamento nesses dados com esse propósito. Como a intenção do trabalho é utilizar características de ambos (dados laser e imagem digital) para identificação e extração dos contornos de telhados, a informação espacial entre esses pontos faz-se necessária. Para tanto, realizou-se o matching (correspondência) entre as imagens de alta resolução e o $\mathrm{MDE}$, por meio da seleção manual de pontos comuns entre a imagem digital e a representação em escala de cinza do $\mathrm{MDE}$, conforme mostrado na Figura 3. Definidos os dois polígonos, calculou-se a matriz de transformação correspondente às duas marcações. Esse cálculo se dá pela distância entre os pontos e pelo ângulo de rotação dos vetores formados pelos pontos. Esses dois parâmetros são aplicados nas transformações geométricas de escala e rotação, conforme representada pela composição de transformações da Equação 2. Essa composição é apresentada também na forma de matriz na Equação 3. O passo seguinte consiste na associação de cada ponto da imagem de alta resolução com seu respectivo ponto no MDE.

$$
M=R(\theta) \cdot S_{x y}
$$

$$
M=\left[\begin{array}{ccc}
\cos (\theta) & -\sin (\theta) & 0 \\
\sin (\theta) & \cos (\theta) & 0 \\
0 & 0 & 1
\end{array}\right]\left[\begin{array}{ccc}
S_{x} & 0 & 0 \\
0 & S_{y} & 0 \\
0 & 0 & 1
\end{array}\right]
$$




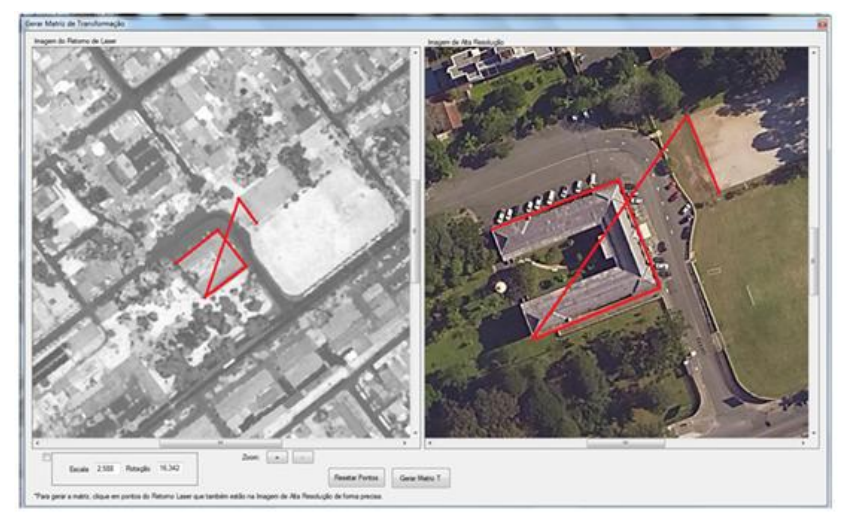

Figura 3. Seleção de pontos em comum entre o MDSn (à esquerda) e a imagem de alta resolução (à direita) para realização do matching.

Ainda foi necessário aplicar um ajuste das informações obtidas da varredura a laser na imagem de alta resolução, dada a diferença de resolução entre as duas fontes de dados. Assim, para que todo o pixel da imagem digital tivesse uma correspondência com o ponto 3D obtido na varredura a laser, foi necessária a replicação de alguns pontos.

\subsubsection{OBTENÇÃO DO MDSn E ÁREAS DE INTERESSE}

A partir do MDE e do MDT é obtido o Modelo Digital de Superfície Normalizado, que consiste na diferença de entre esses dois modelos, com o objetivo de preservar apenas as edificações e demais objetos sobre a superfície, sem a influência do relevo. A partir disso, é definido um limiar referente à altura das edificações para, com isso, obter as regiões de interesse para extração dos telhados. A intenção é eliminar ruas, calçadas, carros, arbustos e outros objetos com altura inferior a de uma construção. $\mathrm{Na}$ Figura 4 são destacadas as regiões cuja altura dos pontos está acima do limiar definido. Nota-se que ruas, calçadas e áreas livres são descartadas das regiões de interesse, mas ainda resta uma grande quantidade de vegetação.

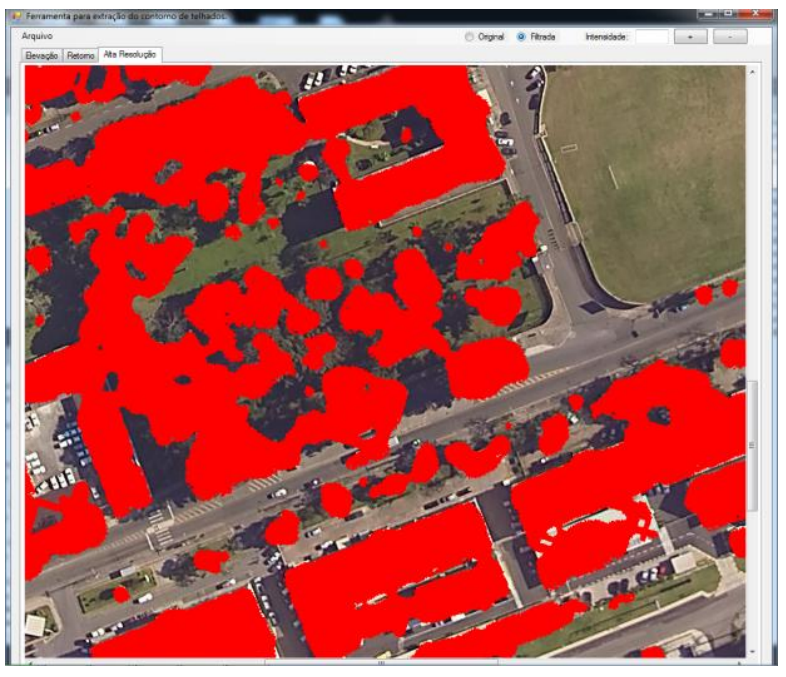

Figura 4. Regiões de interesse iniciais.

\subsubsection{FILTRAGEM USANDO VARIÂNCIA E MORFOLOGIA PARA A ELIMINAÇÃO DE VEGETAÇÃO}

Como a filtragem aplicado ao MDSn com base na altura dos objetos não é suficiente para a resolução do problema, foi aplicada uma filtragem com base na variância da altura dos pontos no intuito de eliminar as regiões de vegetação. Essa filtragem foi proposta visto que as alturas dos pontos de uma árvore apresentam uma disparidade maior quando comparadas às alturas dos pontos de um telhado. O cálculo da variância, conforme Equação 4, consiste em mensurar a 
variação dos indivíduos de um conjunto em relação à sua média aritmética.

Para a obtenção do conjunto de pontos, foi aplicada uma máscara $5 \times 5$ e estabelecidos empiricamente um limiar de variação $s=0.01$ para altura e um limiar $s=4$ para intensidade de retorno. Após o cálculo da variância, a partir dos dados de altura e intensidade, foram mantidas apenas as regiões com variância menor que o limiar definido. Na Figura 5 é mostrado o resultado da aplicação do filtro da variância.

$$
s^{2}(q)=\frac{1}{n-1} \sum_{j=1}^{n}\left(q_{j}-\bar{q}\right)^{2}
$$

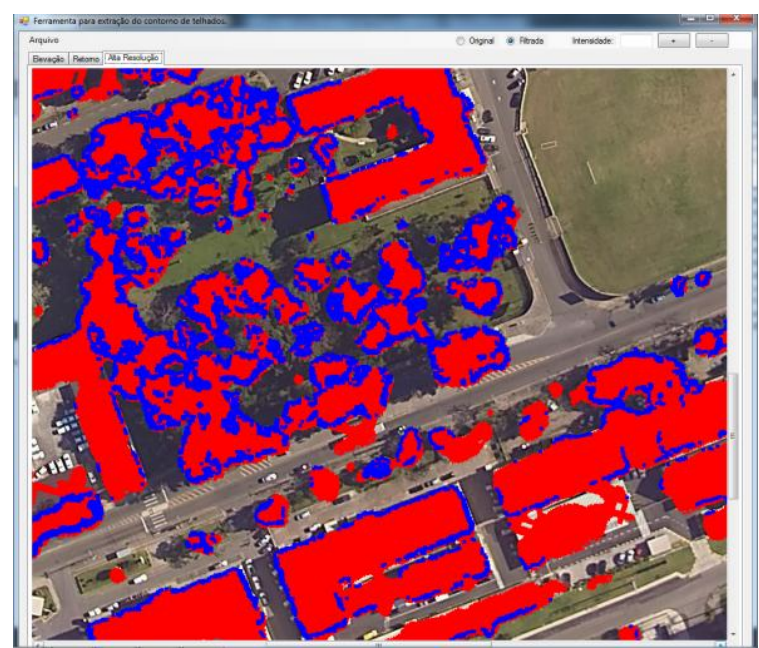

Figura 5. Resultado da aplicação do cálculo da variância sob o conjunto usando uma máscara $5 \times 5$. As áreas em azul representam os conjuntos removidos.

Em seguida, para reduzir ainda mais as áreas de vegetação, foram aplicados os operadores morfológicos abertura e fechamento (GONZALEZ; WOODS, 1993). A abertura e o fechamento são filtros compostos por dois operadores da morfologia matemática: erosão e dilatação. Eles são aplicados na remoção de pequenos objetos e buracos na imagem, por meio da definição de um elemento estruturante, sendo a abertura resultante da aplicação da erosão seguida da dilatação, e o fechamento, da aplicação dos mesmos operadores em ordem contrária. A abertura e o fechamento foram aplicados no resultado do filtro da variância (Figura 7(a)) para remover as pequenas áreas remanescentes dessa filtragem, como mostrado na Figura 7(b).

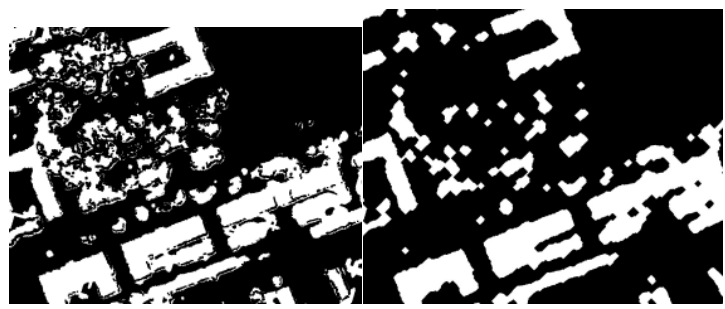

(a)

(b)

Figura 7. Resultado da aplicação da abertura e fechamento nas regiões de interesse. $\mathrm{Na}$ imagem à esquerda (a) tem-se o resultado do filtro da variância e na imagem à direita (b) o resultado da aplicação da abertura e fechamento com elemento estruturante $3 \times 3$.

\subsubsection{SEPARAÇÃO DE TELHADOS USANDO A DETECÇÃO DE BORDAS CANNY E FILTRAGEM POR ÁREA}

Com a abertura e fechamento, alguns detalhes e bordas se fundiram, assim, a solução empregada para resolver esse problema foi subtrair da imagem resultante da abertura e fechamento as bordas obtidas com a aplicação do algoritmo de Canny 
(CANNY, 1986). O algoritmo foi aplicado sobre a imagem em níveis de cinza, gerada a partir da informação de elevação dos pontos da MDE, conforme mostrada na Figura 8(a). Nele são utilizados dois parâmetros (thresh e threshLinking), os quais permitem aumentar ou diminuir o nível de detalhamento e quantidade de bordas extraídas conforme desejado. Os valores dos parâmetros thresh e threshLinking utilizados foram 40 e 120, respectivamente, definidos empiricamente, por meio de testes e análise visual dos resultados. O resultado da extração das bordas do MDSn é mostrado na Figura 8(b). Além disso, por meio de testes, constatou-se que regiões com áreas inferiores a certo valor também poderiam ser descartadas. Isso foi realizado, pois algumas regiões não possuíam dimensões correspondentes a uma edificação. O resultado da separação das regiões de interesse por meio das bordas e da eliminação com base na área é mostrado na Figura 9.

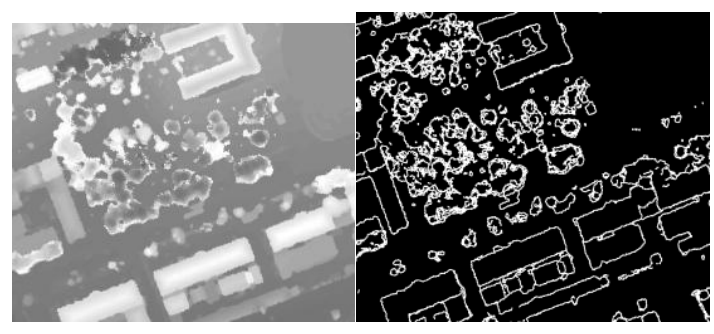

(a)

(b)

Figura 8. Extração das bordas do MDE utilizando a técnica de Canny.

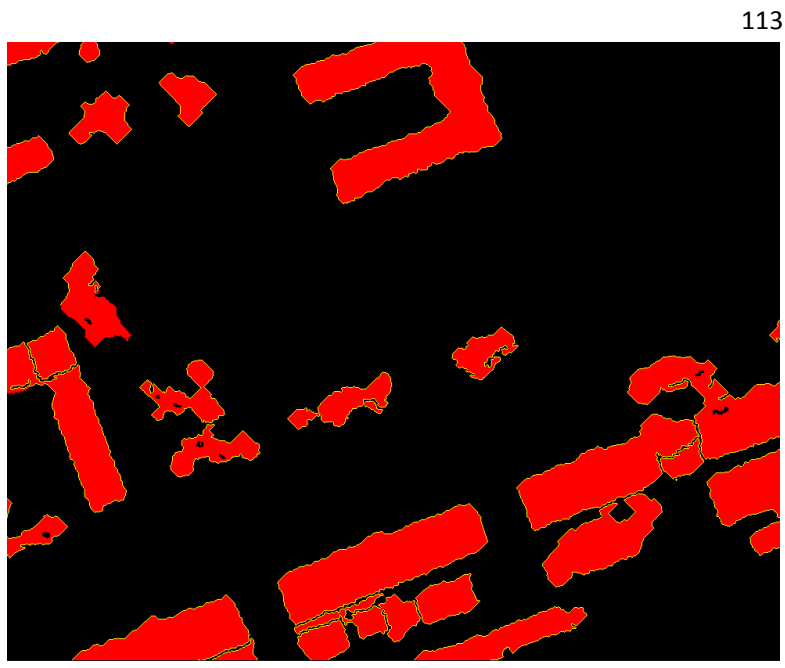

Figura 9. Resultado da separação das regiões usando as bordas e da remoção de áreas inferiores a um limiar (1600 pixels).

\subsubsection{EXTRAÇÃO DOS CONTORNOS DAS REGIÕES DE INTERESSE UTILIZANDO A TÉCNICA DE PERSEGUIÇÃO DE BORDAS.}

Com as regiões de interesse refinadas, é preciso agora extrair os contornos. Para isso, foi utilizada a técnica de perseguição de bordas, ou algoritmo do "ceguinho", que consiste em perseguir uma divisa até encontrar o ponto de início. Para encontrar uma região, a imagem é varrida até que seja encontrada uma divisa, e cada ponto percorrido é guardado em uma lista em ordem, de modo a formar um polígono. Ao final, por se tratarem de várias regiões, uma lista contendo todos os contornos é criada.

\subsubsection{FILTRAGEM DO CONTORNO DOS TELHADOS UTILIZANDO O ALGORITMO DE DOUGLAS-PEUCKER.}

Para criar feições lineares a partir do contorno extraído das regiões de interesse 
foi utilizado o algoritmo Douglas-Peucker (DOUGLAS; PEUCKER, 1973). Esse algoritmo mantém $\mathrm{o}$ ponto de maior distância perpendicular ao segmento definido pelos pontos inicial e final do conjunto de pontos, que é testado a partir de um valor de tolerância T. Na Figura 10 são mostrados os passos para linearizar o contorno. Na Figura 11 é mostrado o resultado obtido com a aplicação do método sobre os contornos extraídos no passo anterior.

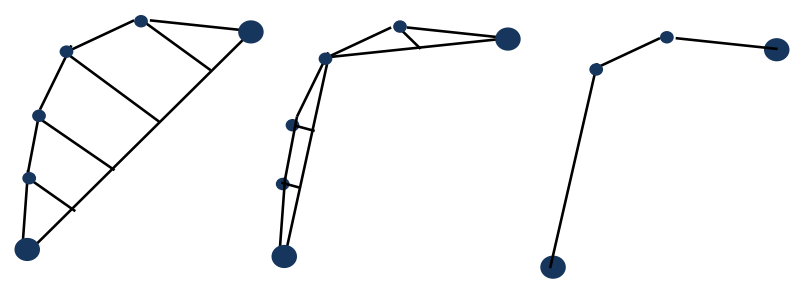

Figura 10. Representação do funcionamento de Douglas-Peucker. A cada iteração pontos do polígono são removidos caso a distância perpendicular do ponto seja menor que a tolerância $T$.

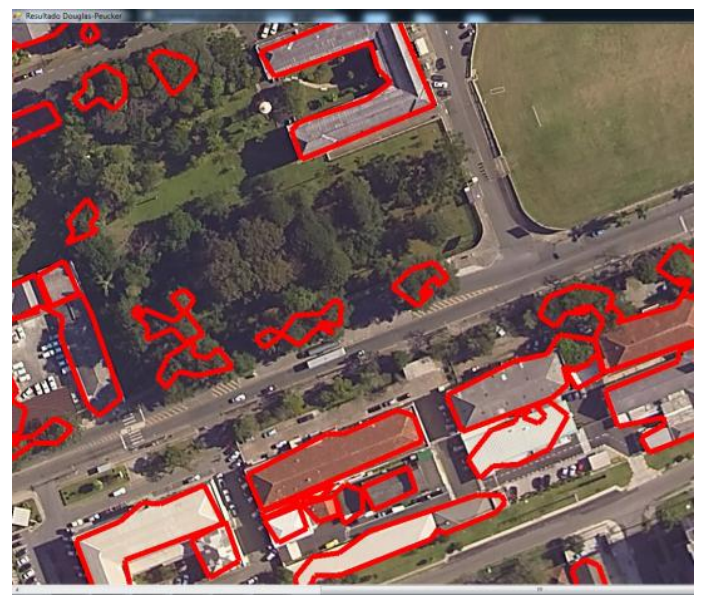

Figura 11. Aplicação do algoritmo de Douglas-Peucker em cada contorno (polígono) armazenado na lista, com T = 9.2.

\subsubsection{FILTRAGEM DE REGIÕES DE VEGETAÇÃO UTILIZANDO A COR.}

As regiões de vegetação na imagem de alta resolução possuem uma coloração característica que não é facilmente encontrada em telhados, e algumas análises podem ser feitas no padrão de coloração dessas áreas trabalhando com os espaços de cores existentes, sendo que nesse caso foi utilizado o espaço de cores RGB. Algumas amostras de pixels das regiões de vegetação nas imagens aéreas disponíveis foram avaliadas, e alguns intervalos de cores RGB foram reconhecidos, os quais são mostrados na Figura 12 (b). Assim, a filtragem foi feita utilizando a regra: $\mathrm{R} / \mathrm{G}<1.12 \mathrm{E} \mathrm{B} / \mathrm{G}<1.05 \mathrm{E}$ $\mathrm{R}+\mathrm{G}+\mathrm{B}<=310$.

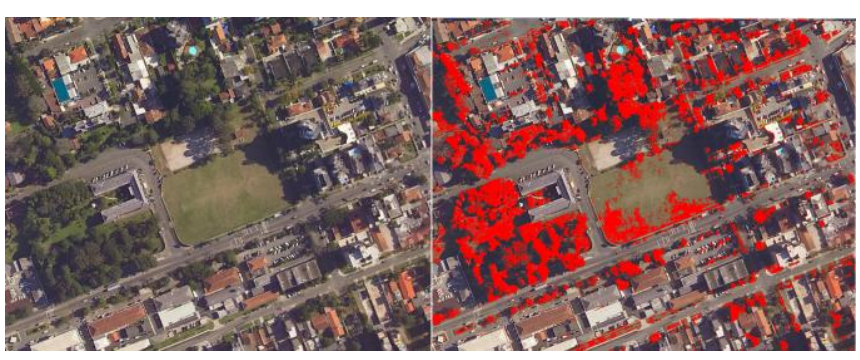

(a)

(b)

Figura 12. Resultado da filtragem da vegetação utilizando a coloração: As regiões em vermelho na imagem (b) representam a vegetação. Foi possível filtrar as regiões de vegetação, mantendo a integridade na maioria dos telhados. 
Mesmo após a aplicação do filtro da variância, ainda restaram algumas áreas de vegetação dentro das regiões de interesse. Para remover essas áreas remanescentes, foi considerado o resultado da filtragem de vegetação pela cor aplicada sobre a imagem de alta resolução. As áreas foram analisadas individualmente, de forma que aquelas com mais de $50 \%$ de sua área com coloração dentro dos parâmetros definidos para vegetação fossem removidas. Na Figura 13 é apresentado o resultado após a remoção dos contornos sobre áreas de vegetação.

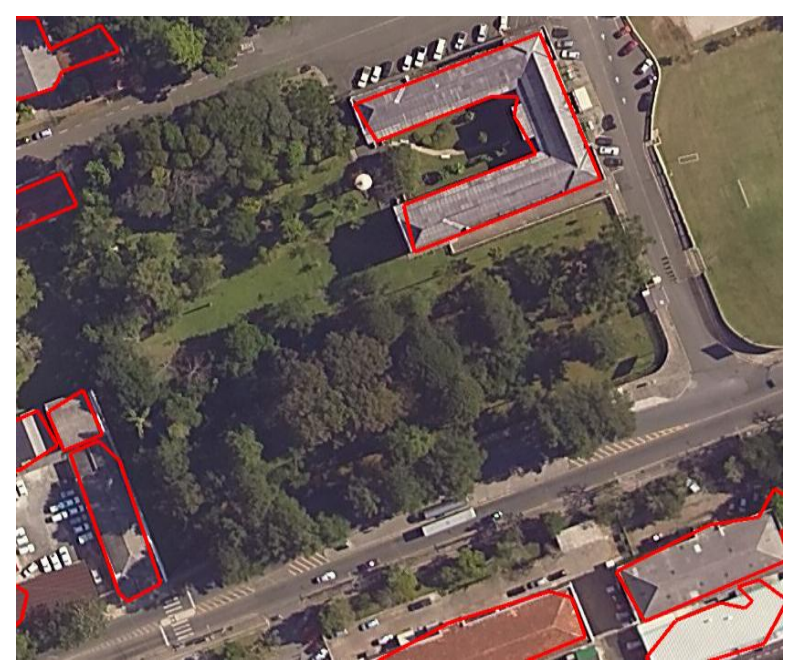

Figura 13. Resultado da remoção de áreas de vegetação.

\section{ANÁLISE DOS CONTORNOS EXTRAÍDOS}

A seguir são mostrados os comparativos entre os contornos extraídos pela ferramenta desenvolvida e o contorno de referência. O contorno de referência foi feito manualmente utilizando a imagem de alta resolução apenas para fins de avaliação dos resultados obtidos. Na Figura 14 são mostrados alguns resultados alcançados na extração de telhados, sendo os segmentos na cor vermelha o resultado obtido com a ferramenta, e em azul o contorno de referência.

Apesar de algumas imperfeições ocasionadas pela resolução da malha e processos de tratamento dos dados, vários contornos foram obtidos satisfatoriamente (Figura 14(a)(d)(e)). Alguns problemas podem ser percebidos na extração dos telhados nos casos em que eles são muito pequenos, nas regiões em que os telhados ficam muito próximos entre si (Figura 14(f)) ou grudados à vegetação (Figura 14(b)(c)).

Foi realizada uma contagem de telhados na área das Figuras 15 e 16, que possuem uma quantidade considerável de edificações, com o objetivo de avaliar a eficiência da metodologia adotada. O resultado da análise pode ser conferido nas Tabelas 2 e 3 . 


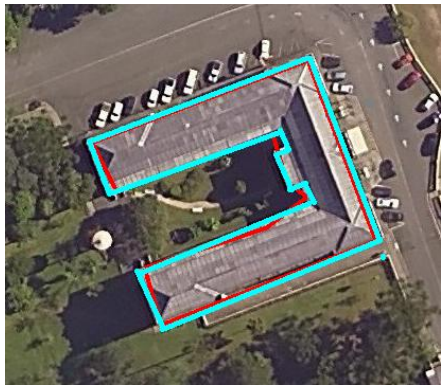

(a)

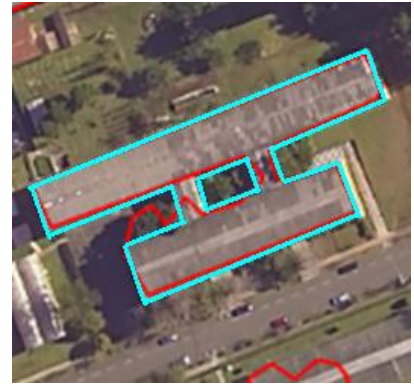

(d)

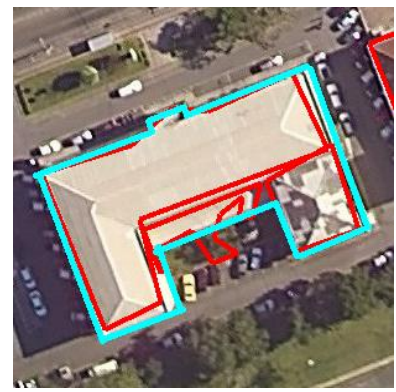

(b)

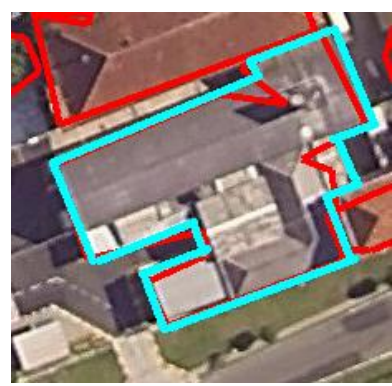

(e)

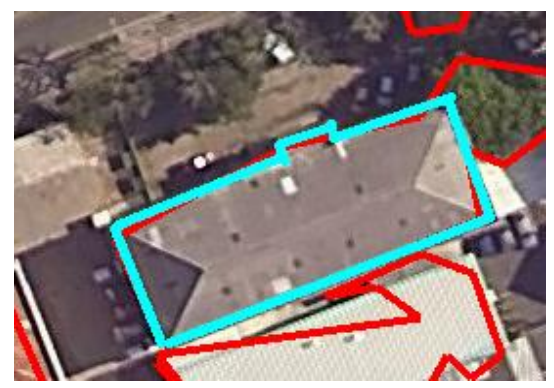

(c)

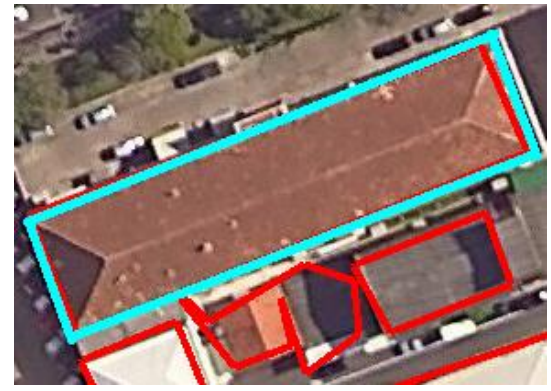

(f)

Figura 14. Resultado da extração de telhados.

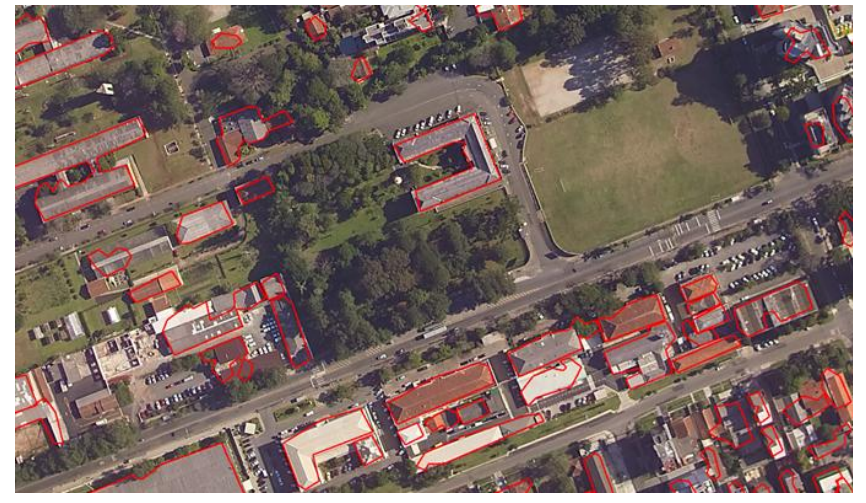

Figura 15. Região de amostra para realizar a quantificação dos telhados encontrados.

Tabela 2. Contagem dos telhados da figura 15, falsos positivos e negativos.

\begin{tabular}{|l|l|l|l|}
\hline & Total de Telhados & Falsos Positivos & Falsos Negativos \\
\hline Quantidade & 66 & 0 & 2 \\
\hline Porcentagem & $100 \%$ & $0 \%$ & $3.03 \%$ \\
\hline
\end{tabular}

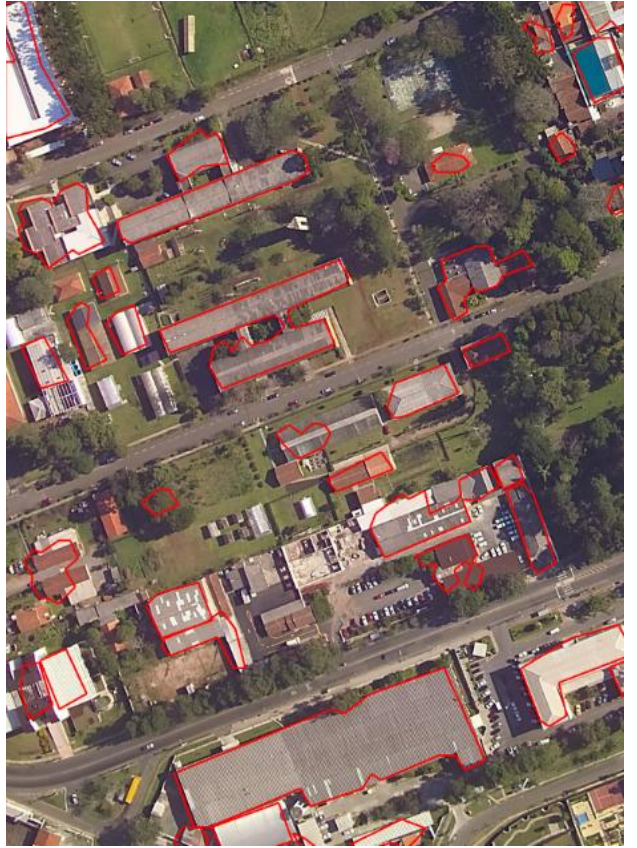

Figura 16. Região de amostra para realizar a quantificação dos telhados encontrados. 
Tabela 3. Contagem dos telhados da figura 16 , falsos positivos e negativos.

\begin{tabular}{|l|l|l|l|}
\hline & Total de Telhados & Falsos Positivos & Falsos Negativos \\
\hline Quantidade & 34 & 1 & 4 \\
\hline Porcentagem & $100 \%$ & $2,9 \%$ & $11,76 \%$ \\
\hline
\end{tabular}

Pode ser verificado que os falsos positivos foram mínimos, enquanto houve um maior índice de falsos negativos. . A maior parte dos falsos negativos ocorre em telhados muito pequenos, pelo fato da malha regular ter uma resolução baixa, por algumas falhas na geração do MDT e também na replicação dos pontos na imagem de alta resolução.

\section{CONSIDERAÇÕES FINAIS}

Comparando os resultados obtidos com vários trabalhos relacionados, percebe-se que cada método desenvolvido possui pontos fracos, e que em algumas situações cada método possui sua vantagem. Com o tempo, tais métodos vão sendo aprimorados, gerando melhores resultados.

A remoção total da vegetação é muito difícil e é um obstáculo em praticamente todos os métodos, principalmente pelo fato de a vegetação possuir variações semelhantes aos telhados em alguns aspectos e, por esse motivo, acabam sendo consideradas como parte das regiões de interesse.

A qualidade dos dados, assim como o método utilizado na geração do modelo digital influencia fortemente nos resultados da extração das feições dos telhados. Podem ser percebidas visualmente algumas deformações nos telhados ocasionadas por processos anteriores que, consequentemente, afetaram alguns resultados negativamente.

As imagens de alta resolução possuem detalhes refinados e informações importantes para serem comparadas às feições extraídas dos dados laser, porém, é muito difícil de realizar essa comparação de maneira automática pelo fato de suas feições serem extremamente complexas.

Caso seja utilizada uma imagem de alta resolução com perspectiva ortogonal aos telhados, e com uma maior densidade de pontos por área dos dados LIDAR, a eficiência do método desenvolvido tenderá a aumentar.

Para trabalho futuro, existe a possibilidade de reconstrução dos contornos, podendo ser utilizada plantas baixas como no trabalho de Park et al. (2006) ou segmentos mais definidos extraídos de imagens de alta resolução. Outros trabalhos importantes e necessários são a associação de terrenos com os respectivos telhados utilizando a informação de georreferenciamento de ambos e a revisão dos métodos de tratamento de dados anteriores à extração do contorno dos telhados, que estão causando falhas no resultado final. 


\section{REFERÊNCIAS}

BURROUGH, P. A. Principles of Geographical Information Systems. 1. ed. New York: Oxford University Press, 1989.

CANNY, J. A computational approach to edge detection. IEEE Trans. Pattern Analysis and Machine Intelligence, v.8, n.6, p.679-698, 1986.

CARDOSO, W. F.; SILVA, F. A.; PAZOTI, M. A. Geração de modelo digital de terreno por meio da filtragem de dados obtidos por varredura a laser utilizando um método baseado no princípio da declividade. Colloquium Exactarum, v. 6, p. 46-64, 2014.

CENTENO, J. A. S.; MITISHITA, E. A. Laser scanner aerotransportado no estudo de áreas urbanas: A experiência da UFPR. In: SIMPÓSIO BRASILEIRO DE SENSORIAMENTO REMOTO, 13., Anais... Florianópolis, Brasil, 21-26 abril 2007, INPE. p. 3645-3652.

DOUGLAS, D.; PEUCKER, T. Algorithms for the Reduction of the Number of Points Required to Represent a Digitized Line or its Caricature, The Canadian Cartographer, vol. 10, n. 2, December 1973, pp.112-122, 1973.

FAZAN, A. J. Extração de contornos de telhado de edifícios no espaço-objeto baseada em snakes e programação dinâmica a partir de TIN/LASER e imagens aéreas de alta resolução. 120p. Tese (Doutorado em Ciências Cartográficas) - Faculdade de Ciências e Tecnologia, Universidade Estadual Paulista, Presidente Prudente, 2011.

FELGUEIRAS, C. A. Modelagem Numérica de Terreno. Relatório Técnico INPE-8081PRE/3896, INPE, São José dos Campos, 2001.

GALVANIN, E. A. S. Extração automática de contornos de telhados de edifícios em um modelo digital de elevação, utilizando inferência bayesiana e campos aleatórios de Markov. 165p. Tese (Doutorado em Ciências Cartográficas) - Faculdade de Ciências e Tecnologia, Universidade Estadual Paulista, Presidente Prudente, 2007.

GONZALEZ, Rafael C; WOODS, Richard E. Digital Image Processing. Reading, Massachusetts: Addison-Wesley, 1993.

MACHADO, A. M. L.; MITISHITA, E. A. Detecção automática de contornos de edificações utilizando imagem gerada por câmara digital de pequeno formato e dados lidar. Boletim de Ciências Geodésicas, v. 12, n. 2, p. 215-233, 2006.

MATIKAINEN, L.; HYYPPÄ J.; HYYPPÄ H. Automatic detection of buildings from laser scanner data for map updating. In: ISPRS WORKSHOP "LASER SCANNING 2005". Proceedings... Enschede, the Netherlands, 2005.

NARDINOCCHI, C.; FORLANI, G.; ZINGARETTI, $P$. Classification and filtering of laser data. In: ISPRS WORKING GROUP III/3 WORKSHOP. Proceedings... Dresden, Germany, 2003.

PARK, J.; LEE, I.; CHOI, Y.; LEE, Y. J. Automatic Extraction of Large Complex Buildings Using Lidar Data and Digital Maps. In: SYMPOSIUM OF ISPRS COMMISSION III PHOTOGRAMMETRIC COMPUTER VISION. Proceedings... Bonn, Germany, 2006.

ROTTENSTEINER, F.; BRIESE, C. A new method for building extraction in urban areas from high-resolution lidar data. In: IAPRSIS, v. XXXIV/3A, Graz, Áustria, 2002. Proceedings... 2002, p. 295-301.

SOHN, G.; DOWMAN, I. J. Building extraction using Lidar DEMs and Ikonos images. In: ISPRS Working Group III/3 Workshop, Dresden, Germany. Proceedings... v. 34, Part 3/W13, pp. 8-10, 2003. 
TARSHA-KURDI, F.; LANDES, T.; GRUSSENMEYER, P.; SMIGIEL, E. New approach for automatic detection of buildings in airborne laser scanner data using first echo only. In: SYMPOSIUM OF ISPRS COMMISSION III PHOTOGRAMMETRIC COMPUTER VISION. Proceedings... Bonn, Germany, v. 36, pp. 25-30, 2006.

TÓVÁRI, D.; PFEIFER, N. Segmentation based robust interpolation - A new approach to laser data filtering. In: ISPRS WORKSHOP "LASER SCANNING 2005". Proceedings... Enschede, the Netherlands, 2005, pp. 79-84.

VOSSELMAN, G.; DIJKMAN, S. 3D building model reconstruction from point clouds and ground plans. In: INTERNATIONAL ARCHIVES OF PHOTOGRAMMETRY AND REMOTE SENSING (IAPRS2001), v. XXXIV-3/W4, Annapolis. Proceedings... 2001, pp. 37-43. 